\title{
WHICH VANCOMYCIN DOSING SCHEDULE FOR SIGNIFICANTLY PRETERM INFANTS?
}

\author{
J. Berrington, G. Gerardos, K. Lekhak
}

Newcastle Neonatal Service, Newcastle Upon Tyne Hospitals NHS Trust, Newcastle Upon Tyne, UK

Background: Coagulaes negative staphylococcal infection is the commonest organism identified in preterms and usually treated with vancomycin. Dosing regimes for significantly preterm infants vary between reference sources and lack supportive data.

Aims: To assess vancomycin levels in infants $<29$ weeks (or $1.2 \mathrm{kgs}$ ) receiving their first vancomycin course, and analyse levels acheieved in relation to regime received.

Methods: An audit of first vancomycin levels acheived was undertaken and levels were categorised as adequate $(5-20 \mathrm{mg} / \mathrm{l})$, sub-therapeutic $(<5 \mathrm{mg} / \mathrm{l})$ or high $(>20 \mathrm{mg} / \mathrm{l})$. The proportion of infants with therapeutic levels resulting from dose regimes from 3 common sources $(\mathrm{BNFc}$, Northern Neonatal Formulary(5) and AAP guidance) was analysed.

Results: The Northern Neonatal Formulary achieved the highest proportion of adequate levels when given as per recommendation. BNFc/AAP guidance resulted in higher proportions of subtherapeutic levels when given as per their recommended dosing.

\begin{tabular}{|c|c|c|c|c|c|c|c|c|c|}
\hline \multirow{3}{*}{$\begin{array}{l}\text { Guidance as per } \\
\rightarrow \% \text { (n) with trough }(\rightarrow=5- \\
20 \mathrm{mg} / 1, \downarrow=<5 \mathrm{mg} / 1, \uparrow=>20 \mathrm{mg} / 1\end{array}$} & \multicolumn{9}{|c|}{ Dose in relation to recomendation } \\
\hline & \multicolumn{3}{|c|}{ As recomended } & \multicolumn{3}{|l|}{ Lower } & \multicolumn{3}{|l|}{ Higher } \\
\hline & $\rightarrow$ & $\downarrow$ & $\uparrow$ & $\rightarrow$ & $\downarrow$ & $\uparrow$ & $\rightarrow$ & $\downarrow$ & $\uparrow$ \\
\hline NNF5 & $100(2)$ & - & - & $61(7)$ & $38(5)$ & - & - & - & - \\
\hline $\mathrm{BNFc}$ & $58(7)$ & $42(5)$ & - & $100(1)$ & - & - & $100(2)$ & |- & - \\
\hline AAP & $58(7)$ & $42(5)$ & - & $100(1)$ & - & - & $100(2)$ & |- & - \\
\hline
\end{tabular}

[Vancomycin levels by regime]

Conclusion: Guidance in the NNF5 appears most suited to significantly preterm (or $<1.2 \mathrm{~kg}$ ) infants. 